\title{
Active Contours based Object Detection and Extraction using SPF Parameter
}

\author{
Savan Kumar Oad \\ EC Dept., GGITM \\ Raisen Road, Anand Nagar \\ Bhopal, MP-462021
}

\author{
Karuna Markam \\ EC Dept., BUUIT \\ Hoshangabad Road \\ Bhopal, MP-462026
}

\author{
Aditya Kumar Bhatt \\ EX Dept., GGITM \\ Raisen Road, Anand Nagar \\ Bhopal, MP-462021
}

\begin{abstract}
In this paper, we propose a new region-based Active Contour Model (ACM) that employs signed pressure force (SPF) as a level set function. Further, a flood fill algorithm is incorporated along with SPF function for robust object extraction. Signed pressure force (SPF) parameters, is able to control the direction of evolution of the region. The proposed system shares all advantages of the $\mathrm{C}-\mathrm{V}$ and GAC models. The proposed ACM has an additional advantage i.e. of selective local or global segmentation. Flood Fill framework is employed for retrieving the object upon successful detection in the image. In addition, the computer simulation results show that the proposed system could address object detection within an image and its extraction with highest order of efficiency.
\end{abstract}

\section{General Terms}

Pattern Recognition, Security, Image Segmentation

\section{Keywords}

Image segmentation, signed pressure force parameters, flood fill algorithm, threshold segmentation

\section{INTRODUCTION}

In current digital era, image segmentation has established itself as an active research area in the field of computer vision and image analysis. One of the major problems that we come across during the image processing analysis is to extract the region of interest (i.e. segmentation). In general, segmentation is a process of partitioning a digital image into multiple regions (sets of pixels), according to some homogeneity criterion. Extensive study for segmentation has been made and many techniques have been proposed. There are a wide variety of approaches that are used for segmenting an image of which ACM [2] is most successful approach.

Active contour models have widely been used for image segmentation and level set theory in active contours increases the flexibility. The basic idea in active contour models (or snakes) is to evolve a curve, in order to detect objects in that image. Generally ACMs can be categorized as Edge based models [2,4,5,7,11,17,18,20] and Region based models $[6,8,9,12,14,15,16]$. The Edge based models rely on a gradient based stopping function to stop the curve evolution whereas Region-based models utilize the image statistical information to construct constraints and can successfully segment objects with weak boundaries. Most popular edge based model is GAC model [4, 5]. GAC model utilizes image gradient to construct an edge stopping function (ESF) to stop the contour evolution on the object boundaries. The GAC model can only extract the object when the initial contour surrounds its boundary, and it cannot detect the interior contour without setting the initial one inside the object. Thus, we can say that the GAC model possesses local segmentation property which can only segment the desired object with a proper initial contour.

In addition, researchers are conducting extensive research in designing an active contour model without edge stopping function, i.e., a model that does not use the gradient of the image for the stopping process. These models are generally known as Region based models. One of the most popular region based model is the $\mathrm{C}-\mathrm{V}$ model [6] which utilizes the statistical information inside and outside the contour to control the evolution. The $\mathrm{C}-\mathrm{V}$ model [6] has the global segmentation property to segment all objects in an image. However, the $\mathrm{C}-\mathrm{V}$ model does not work well for the images with intensity in homogeneity.

In this paper, a new region-based Active Contour Model (ACM) is proposed which shares the advantages of the $\mathrm{C}-\mathrm{V}$ and GAC models. A new level set function is designed in this paper which is known as Signed Pressure force (SPF) function [10] and a region growing algorithm [3] known as Flood Fill algorithm is also applied with SPF function in this paper. The proposed SPF function has opposite signs, so the contour can shrink when it is outside the object or expand when inside the object. In addition it incorporates a unique aspect of selective local or global segmentation, which can segment the desired objects as well as accurately segment all the objects with interior and exterior boundaries. After detection of desired object, within an image, it is to be retrieved from the image and for this purpose Flood fill algorithm is employed.

The rest of the paper is organized as follows. In section 2, we discuss the existing works in the field of image segmentation with reference to the object detection and extraction. In addition, we present the merits and demerits of these systems in brief. Section 3 deals with the proposed model that counters the demerits of the existing systems while offering the merits. In section 4, we present the simulation results and analysis of the proposed system. And section 5 provides the conclusion of the paper.

\section{BACKGROUND}

Chan-Vese model [6] is the first region based geometric active contour model which can be seen as a special case of the Mumford -Shah Problem [9]. The evolution function given by Chan- Vese is as follows:

$\left.\frac{\partial \phi}{\partial t}=\delta_{\varepsilon}(\phi)\left[\lambda_{1}\left(I-c_{1}\right)^{2}-\lambda_{2}\left(I-c_{2}\right)^{2}\right]+\mu . k+v\right]$

Where $\mu \geq 0, v \geq 0, \lambda_{1}>0, \lambda_{2}>0$ are fixed coefficients, $c_{1}$ and $c_{2}$ are two target values that are mean intensities of the image areas inside and outside the contours, respectively, $\kappa$ is the 
mean curvature of the contours and is the $\delta_{\varepsilon}$ is the Delta function.

For a given image $\mathrm{I}$ in domain $\Omega$, the $\mathrm{C}-\mathrm{V}$ model is formulated by minimizing the following energy functional:

$$
\begin{aligned}
& \mathbf{E}_{\mathrm{CV}}=\lambda_{1} \int_{\text {inside }(C)}\left|\mathbf{I}(\mathbf{x})-\mathbf{c}_{1}\right|^{2} \mathbf{d x}+\lambda_{2} \int_{\text {outside }(C)} \mid \mathbf{I}(\mathbf{x})- \\
& \left.\mathbf{c}_{2}\right|^{2} \mathbf{d x}, \mathbf{x} \in \Omega
\end{aligned}
$$

Where $c_{1}$ and $c_{2}$ are two constants which are the average intensities inside and outside the contour, respectively, with the level set method, we assume.

$$
\left\{\begin{array}{c}
\mathrm{C}=\{x \in \Omega: \phi(x)=0\} \\
\text { Inside }(\mathrm{C})=\{x \in \Omega: \phi(x)>0\} . \\
\text { Outside (C) }\{x \in \Omega: \phi(x)<0\} .
\end{array}\right.
$$

By minimizing Eq. (3.21) we solve $c_{1}$ and $c_{2}$ as follows:

$$
\begin{aligned}
& \mathrm{C}_{1}(\phi)=\frac{\int_{\Omega} I(x) \cdot H(\phi) d x}{\int_{\Omega} H(\phi) d x} \\
& \mathrm{C}_{2}(\phi)=\frac{\int_{\Omega} I(x) \cdot(1-H(\phi) d x}{\int_{\Omega}(1-H(\phi) d x}
\end{aligned}
$$

$H(\phi)$ is the Heaviside function and $\delta_{\varepsilon}(\phi)$ is the Dirac function. Generally, the regularized versions are selected as follows:

$$
\left\{\begin{array}{c}
H(\phi)=\frac{1}{2}\left(1+\frac{2}{\pi} \arctan \left(\frac{z}{\varepsilon}\right)\right), \\
\delta_{\varepsilon}(\phi)=\frac{1}{\pi} \frac{\varepsilon}{\varepsilon^{2}+z^{2}}, \phi \varepsilon R
\end{array}\right.
$$

\subsection{The GAC Model}

One of the most successful edge based geometric active contour model is the Geodesic Active Contour (GAC) model $[4,5]$. The GAC model proposed by Caselles et al. and Malladi et al., as well as the Snake model, are EGAC models, which means that the contour evolution speed is based on the edge information in images. Let $\Omega$ be a bounded open subset of $\mathrm{R}^{2}$ and I: $[0, \mathrm{a}] \mathrm{X}[0, \mathrm{~b}] \rightarrow \mathrm{R}^{+}$be a given image. Let $\mathrm{C}(\mathrm{q})$ : $[0,1] \rightarrow R^{2}$ be a parameterized planar curve in $\Omega$. The GAC model is formulated by minimizing the following energy functional:

$$
\begin{aligned}
& \mathrm{E}^{\mathrm{GAC}}(\mathrm{C})=\int_{0}^{1} g(|\nabla I(C(q))|)\left|c^{\prime}(q)\right| d q \\
& \mathrm{C}_{1}=\mathrm{g}(|\nabla I|) \mathrm{k} \overrightarrow{\mathrm{N}}-(\nabla g \cdot \vec{N}) \overrightarrow{\mathrm{N}}
\end{aligned}
$$

Where $\mathrm{k}$ is the curvature of the contour and $\overrightarrow{\mathrm{N}_{1}}$ s the inward normal to the curve. Usually a constant velocity term $\alpha$ is added to increase the propagation speed. Then Eq. (6) can be rewritten as

$$
\mathrm{C}_{1}=\mathrm{g}(|\nabla I|)(\mathrm{k}+\alpha) \overrightarrow{\mathrm{N}}-(\nabla g \cdot \vec{N}) \overrightarrow{\mathrm{N}}
$$

The corresponding level set formulation is as follows:

$\frac{\partial \phi}{\partial t}=\mathbf{g}|\nabla \phi|\left(\operatorname{div}\left(\frac{\nabla \phi}{|\nabla \phi|}\right)+\alpha\right)+\nabla g \cdot \nabla \phi$

Where $\alpha$ is the balloon force, which controls the contour shrinking or explanation with respect to the object that needs to be detected and extracted.

\subsection{Flood Fill Algorithm}

Flood Fill method is used to retrieve the selected object in an image, it is also known as region growing or region marking technique. Region growing [3] is a simple region-based image segmentation method that is classified as a pixel based image segmentation method since it involves the selection of initial seed points. In the search for color regions, the most important tasks are to find out which pixels belong to which regions how many regions are in the image and where these regions are located. These steps usually take place as part of a process called region labeling or region coloring. During this process neighboring pixels is pieced together in a stepwise manner to build regions in which all pixels within that region are assigned a unique number ("label") for identification. One efficient fast method is region marking through flood filing in which a region is filled in all directions starting from a single point or "seed" within the region. We must first settle on either the 4 or 8 -connected definition of neighborhood for determining when two pixels are "connected" to each other, since under each definition we can end up with different results.

\section{PROPOSED MODEL}

In this section, we have provided the detail analysis regarding the limitations of the existing methods that are presented in the earlier section. Further, we propose our method that has similar advantages and also fixes the drawbacks of previous methods. When background is clean and clear algorithm discussed previously works fine. Problems occurs when the background is noisy and interior intensities are not homogeneous and also with images having weak edges or without edges.

In order to counter the limitations, we employed a new level set method that is based on Signed Pressure Force (SPF) parameter is proposed. This parameter improves the traditional level set methods as the calculation of SDF and reinitialization [13] is not required. Initially level set function is penalized to be binary, and then a Gaussian filter is used to regularize to cover the entire contour. It is a well known fact that the Gaussian filter can make the evolution more stable [1]. In addition, this model is incorporated with flood fill algorithm to extract the detected object. The proposed model has a property that makes it suitable for both selective local or global segmentation, which can not only extract the desired objects, but also accurately extract all the objects with interior and exterior boundaries.

\subsection{SPF Paramęter}

Region information can be Using calculation of variation [19], we could get an active contour; both to noise and to we robustness of parametric active contours formulations that have incorporated region information can all be written in the following way:

$$
\left.\alpha X_{t}=\left[\alpha(s) X_{s}\right]_{s}-\left[\beta(s) X_{s s}\right]_{s s}+w_{R} * R(X) \cdot 1+1 / P\right)+F_{\text {ext }}(X)
$$

Where, $\mathrm{R}(\mathrm{x})$ is a region function and $\omega_{\mathrm{R}}$ is a positive weighting paramete 3 . 20 re region function is derived from the image and (for the sake of concreteness) has values in the range $[-1,1]$. The region function modulates the sign of the pressure forces used so that the contour shrinks when it is outside the object of interest and expands when it is inside the object. These external forces are defined and controlled by signed pressure forces [10]. The SPF parameter has opposite signs around the object boundary, so the contour can shrink 
when it is outside the object or expand when inside the object. SPF function as formulated in [1] is given as:

$\operatorname{Spf}(I(x))=\frac{I(x)-\frac{c 1+c 2}{2}}{\max \left(\left|I(x)-\frac{c 1+c 2}{2}\right|\right)} \times \varepsilon \Omega$

Where, $c_{1}$ and $c_{2}$ are defined by the Eqs. (3) and (4), respectively. The significance of Eq. (10) can be explained as follows: Refer to Fig.1, we assume that the intensities inside and outside the object are homogeneous. It is intuitive that $\operatorname{Min}(I(x)) \leq \mathrm{C}_{1} \mathrm{C}_{2} \leq \operatorname{Max}(I(\mathrm{x}))$, and the equal signs cannot be obtained simultaneously wherever the contour is. Hence, there is

$\operatorname{Min}(I(x))<\frac{c 1+c 2}{2}<\operatorname{Max}(I(x)) \times \varepsilon \Omega$

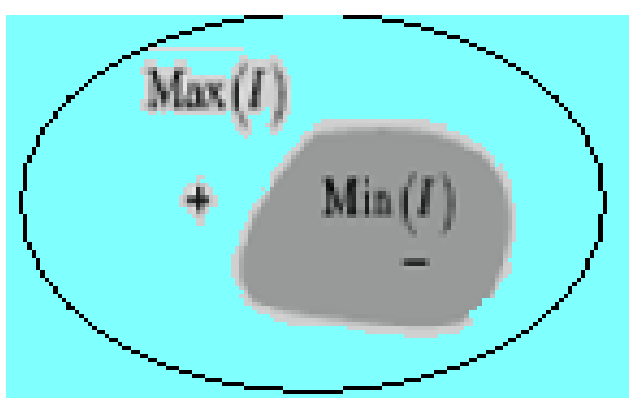

Figure.1 The signs of the SPF function inside and outside the object are opposite

Obviously, the signs of the SPF function in Eq. (10) are identical to what Fig. 1 show. Substituting the SPF function in Eq. (10) for the ESF in Eq. (8), the level set formulation of the proposed model as given in [1] is as follows:

$\frac{\partial \phi}{\partial t}=\operatorname{spf}(I(\mathbf{x}))\left(\operatorname{div}\left(\frac{\nabla \phi}{\mid \nabla \phi}\right)+\propto\right)|\nabla \phi|+\nabla \operatorname{spf}(I(x)) \nabla \phi \ldots(11)$

In addition, the effect of the term " $\nabla s p f(I(x)) \nabla \phi$ " in Eq. (11) can also be ignored, because our model utilizes the statistical information of regions, which has a larger capture range and capacity of anti-edge leakage. Finally, the level set formulation of the proposed model can be written as follows:

$\frac{\partial \phi}{\partial t}=\operatorname{spf}(I(\mathbf{x})) \cdot \alpha|\nabla \phi|, \quad x \varepsilon \Omega$

\subsection{Algorithm}

1. Select input image from database.

2. Define initial level set function $\phi$ to be binary function as :

$\phi(\mathbf{x}, \mathbf{t})= \begin{cases}-\gamma & : \mathrm{x} \text { is in inside domain } \\ \gamma: & \mathrm{x} \text { is in outside domain } \\ 0 & : \mathrm{x} \text { is lies on the boundary. }\end{cases}$

3. Compute parameter as : $\mathrm{C}_{1}(\phi)$ and $\mathrm{C}_{2}(\phi)$

4. Expansion or shrinkage of level set function $\phi$ according to energy minimization or maximization.

5. Using Gaussian filter regularize the level set function.
6. If level set function converges then stop otherwise go to step 3 .

7. If it converges select a seed pixel within object contour.

8. Algorithm for flood fills.

\subsection{Flow Chart}

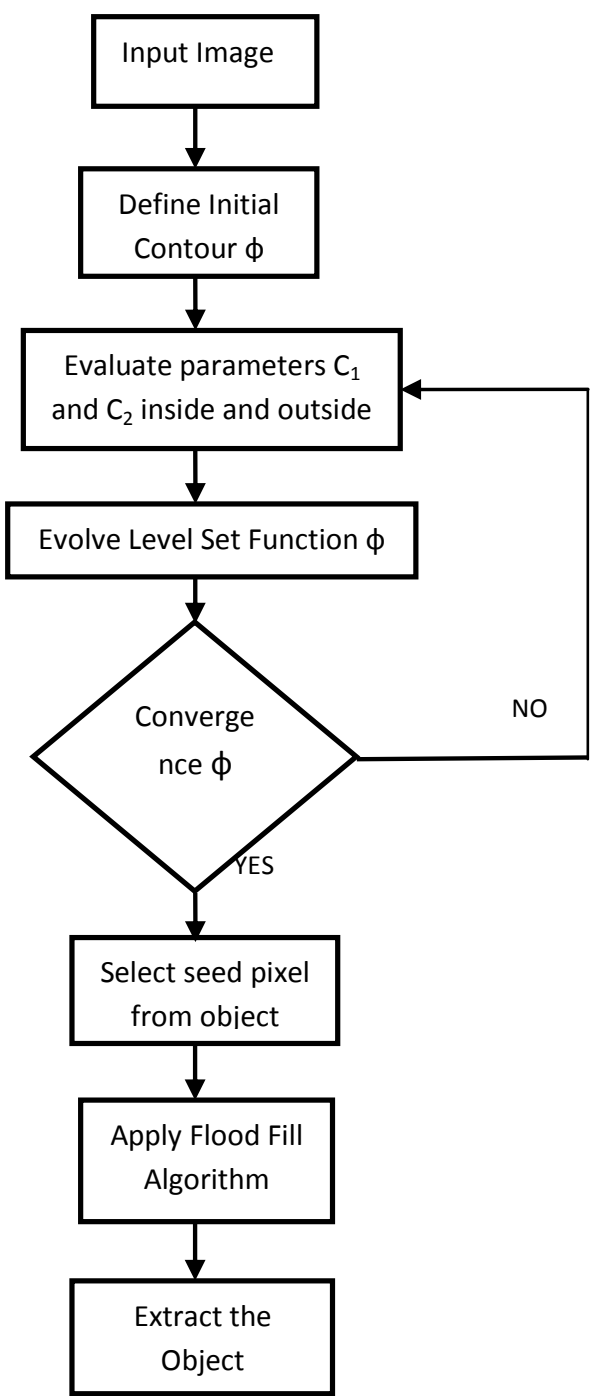

Figure.2 The flow chart of the proposed system

\section{COMPUTER SIMULATIONS}

This section is dedicated to presenting the results of the SPF based segmentation technique as well as image extraction using Flood fill algorithm. Comparison results of segmentation techniques, discussed in section 2, with SPF based ACM are also discussed. We compare the active contour model based on the SPF function explained in previous chapter and the $\mathrm{C}-\mathrm{V}[6]$ and $\mathrm{GAC}[4,5]$ model (having traditional level set formulation). The proposed method applied to a set of real images and in all of them the main object can be found with acceptable amount of error. The initial level set is the square enclosing the image. So there is no need for specific initialization for every single object.

Fig. 3 shows the segmentation results of a synthetic image with objects having weak edges. Fig. 3(a) shows the input 
image to our model. The Fig.3 (b) shows the segmentation result, it is the closed contour obtained by our model and the Fig.3(c) shows the result of our model i.e. objects extraction from input image.

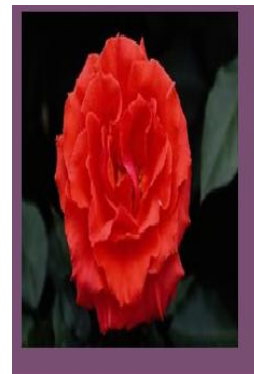

(a)

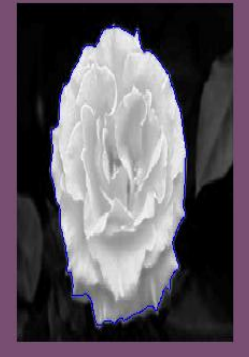

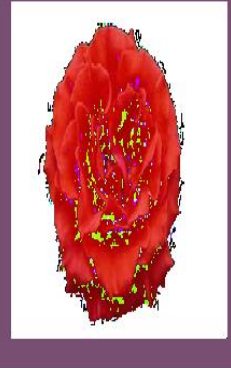

Figure.3 Experiment for a synthetic image (a) Input Image. (b) Object detection using SPF function (closed contour) (c) Object extraction

Fig. 4 and Fig. 5 shows the segmentation results of a galaxy image with some stars around it. Fig. 4(a) \&5(a) shows the input image to our model. The Fig.4(b)\& 5(b) shows the segmentation result, it is the closed contours obtained by our model and the Fig.4(c)\& 5(c) shows the result of our model i.e. object extraction from input image.

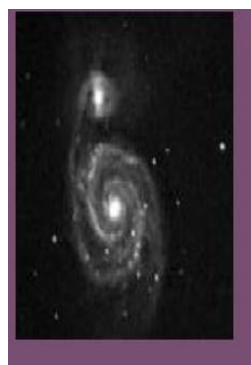

(a)

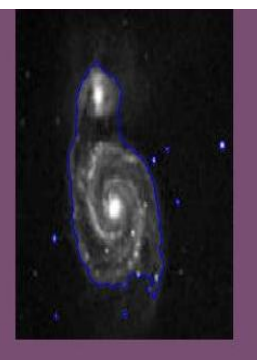

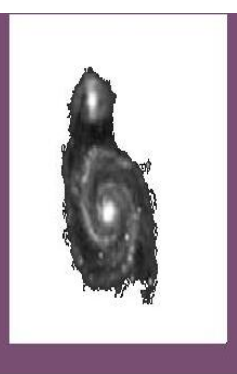

Figure.4 Experiment for a constellation image (a) Input Image.

(b) Object detection using SPF function (closed contours) (c) Object extraction

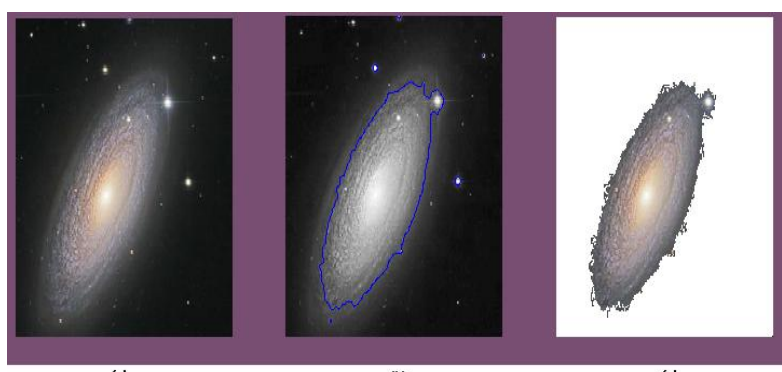

(a)

(c)

Figure.5Experiment for a galaxy image (a) Input Image (b) Object detection using SPF function (closed contours) (c) Object extraction

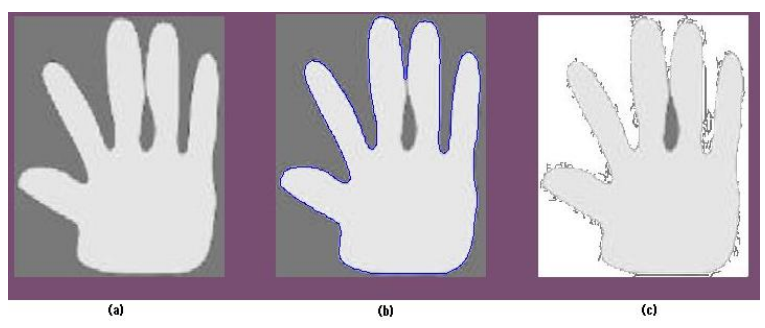

Figure.6Segmentationof the hand phantom using SPF (a) Input Image (b) Object detection using SPF function (closed contours) (c) Object extraction.
Fig. 6 shows the segmentation results of a image. Fig. 6(a) shows the input image to our model. The Fig.6 (b) shows the segmentation result, it is the closed contour obtained by our model and the Fig.6(c) shows the result of our model i.e. objects extraction from input image.

Fig. 7 shows the segmentation results of a synthetic image with objects having weak edges and interior holes. The GAC model with the traditional level set method is used in the comparison. Fig. 7(a) shows the initial contour of our model. The Fig.7 (b) shows the segmentation result of the GAC model. The Fig.7(c) shows the result of our model and the Fig.7 (d) shows the object extraction using our model.

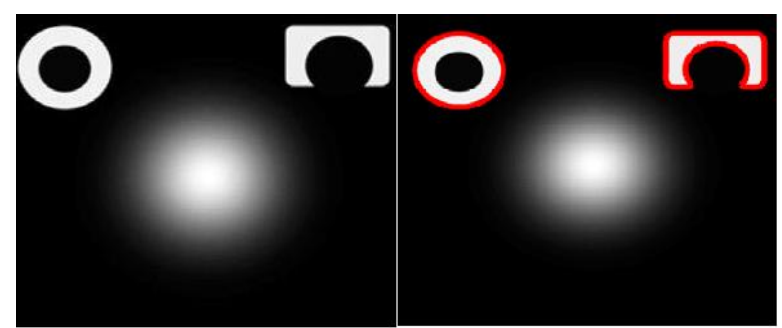

(a)

(b)
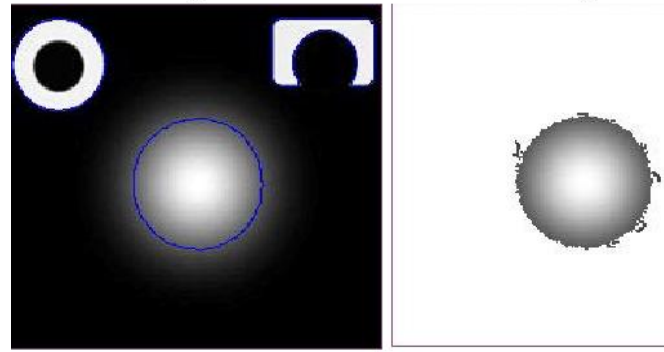

Figure.7Experiments for a synthetic image (a) Input image (b) Result of the GAC model [1]. (c) Result of our method (d) Object extraction.

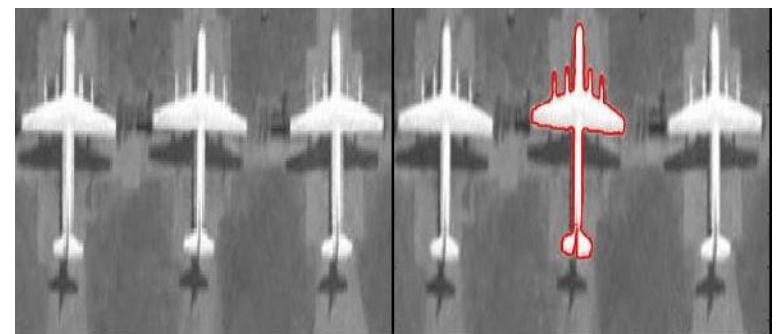

(a)

(b)

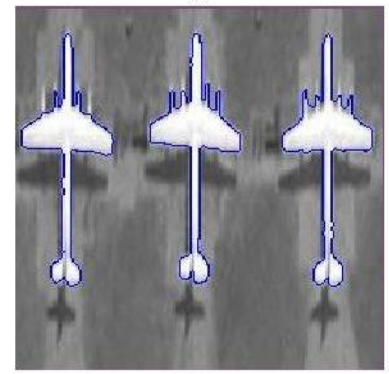

(c)

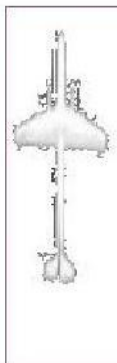

(d)

Figure.8Global segmentation property (a) Input image (b) Result of the C-V model [1]. (c) Result of our method (d) Object extraction.

Fig. 8 demonstrates the global segmentation property of our method. If initial contour is far from the desired object then it is difficult to detect it.Fig.8 (a) shows the input image having 
three objects in it. In case of $\mathrm{C}-\mathrm{V}$ model if initial contour is nearer to middle object then it will detect only middle object and fails to extract all the objects as shown in Fig.8 (b). Whereas our method could accurately detect all the objects, as shown in the Fig.8(c) and any of the three objects can be extracted from the image. Fig.8 (d) shows the extraction of left object of input image.

\section{CONCLUSION}

We have proposed a new image segmentation technique, which can produce desired segmentation outputs on difficult image segmentation problems where traditional segmentation methods cannot create satisfying results. We have developed a fast and efficient model for object detection and extraction and it has both local and global segmentation property. The mathematical implementation of our active contour is achieved by means of Signed Pressure (SPF) Function as a level set function [1] and Flood Fill algorithm as a region growing method [3] for object extraction. By introducing contours as a SPF function, splitting a contour into multiple contours is possible which provide flexibility in the use of active contours. Our experimental results showed clearly that the proposed active contour model based on the SPF function shares the advantages of both GAC $[4,5]$ and $\mathrm{C}-\mathrm{V}$ models [6].

\section{REFERENCES}

[1] K. Zhang, H. Song, W. Zhou, Active contours with selective local and global segmentation: A new formulation and level set method, Image and Vision 28 (2010), pp. 668-676, ELSEVIER.

[2] M. Kass, A. Witkin, D. Terzopoulos, Snakes: active contour models, International Journal of Computer Vision 1 (1988) 321-331.

[3] J. Fan, G. Zeng, M. Body and M.S. Hacid, "Seeded region growing: An extensive and comparative study", Pattern Recognition Letters, Volume 26, Issue 8, pp. 1139-1156, 2005.

[4] V. Caselles, R. Kimmel, G. Sapiro, Geodesic active contours, in: Processing of IEEE International Conference on Computer Vision'95, Boston, MA, 1995, pp. 694-699.

[5] V. Caselles, R. Kimmel, G. Sapiro, Geodesic active contours, International Journal of Computer Vision 22 (1) (1997) 61-79.

[6] T. Chan, L. Vese, Active contours without edges, IEEE Transaction on Image Processing 10 (2) (2001) 266-277.

[7] G.P. Zhu, Sh.Q. Zhang, Q.SH. Zeng, Ch.H. Wang, Boundary-based image segmentation using binary level set method, Optical Engineering 46 (2007) 050501.
[8] J. Lie, M. Lysaker, X.C. Tai, A binary level set model and some application to Munford-Shah image segmentation, IEEE Transaction on Image Processing 15 (2006) 1171-1181

[9] D. Mumford, J. Shah, Optimal approximation by piecewise smooth function and associated variational problems, Communication on Pure and Applied Mathematics 42 (1989) 577-685.

[10] C.Y. Xu, A. Yezzi Jr., J.L. Prince, On the relationship between parametric and geometric active contours, in: Processing of 34th Asilomar Conference on Signals Systems and Computers, 2000, pp. 483-489.

[11] C.M. Li, C.Y. Xu, C.F. Gui, M.D. Fox, Level set evolution without re-initialization: a new variational formulation, in: IEEE Conference on Computer Vision and Pattern Recognition, San Diego, 2005, pp. 430-436.

[12] C.M. Li, C. Kao, J. Gore, Z. Ding, Implicit active contours driven by local binary fitting energy, in: IEEE Conference on Computer Vision and Pattern Recognition, 2007.

[13] S. Osher, R. Fedkiw, Level Set Methods and Dynamic Implicit Surfaces, Springer-Verlag, New York, 2002.

[14] A. Tsai, A. Yezzi, A.S. Willsky, Curve evolution implementation of the Mumford-Shah functional for image segmentation, denoising, interpolation, and magnification, IEEE Transaction on Image Processing 10 (2001) 1169- 1186.

[15] L.A. Vese, T.F. Chan, A multiphase level set framework for image segmentation using the Mumford-Shah model, International Journal of Computer Vision 50 (2002) 271 293.

[16] R. Ronfard, Region-based strategies for active contour models, International Journal of Computer Vision 46 (2002) 223-247.

[17] N. Paragios, R. Deriche, Geodesic active regions and level set methods for supervised texture segmentation, International Journal of Computer Vision 46 (2002) 223247.

[18] C. Xu, J.L. Prince, Snakes, shapes, and gradient vector flow, IEEE Transaction on Image Processing 7 (1998) 359-369.

[19] G. Aubert, P. Kornprobst, Mathematical Problems in Image Processing: Partial Differential Equations and the Calculus of Variations, Springer, New York, 2002.

[20] A. Vasilevskiy, K. Siddiqi, Flux-maximizing geometric flows, IEEE Transaction on Pattern Analysis and Machine Intelligence 24 (2002) 1565-1578 